\title{
A semigroup related to a convex combination of boundary conditions obtained as a result of averaging other semigroups
}

\author{
JACEK BANASIAK AND ADAM BOBROWSKI
}

\begin{abstract}
Let $\alpha$ be a bounded linear operator in a Banach space $\mathbb{X}$, and let $A$ be a closed operator in this space. Suppose that for $\Phi_{1}, \Phi_{2}$ mapping $D(A)$ to another Banach space $\mathbb{Y}, A_{\mid \operatorname{ker} \Phi_{1}}$ and $A_{\mid \operatorname{ker} \Phi_{2}}$ are generators of strongly continuous semigroups in $\mathbb{X}$. Assume finally that $A_{\mid \text {ker } \Phi_{\mathrm{a}}}$, where $\Phi_{\mathrm{a}}=\Phi_{1} \alpha+\Phi_{2} \beta$ and $\beta=I_{\mathbb{X}}-\alpha$, is a generator also. In the case where $\mathbb{X}$ is an $L^{1}$-type space, and $\alpha$ is an operator of multiplication by a function $0 \leq \alpha \leq 1$, it is tempting to think of the later semigroup as describing dynamics which, while at state $x$, is subject to the rules of $A_{\mid} \mid \operatorname{ker} \Phi_{1}$ with probability $\alpha(x)$ and is subject to the rules of $A_{\mid \text {ker } \Phi_{2}}$ with probability $\beta(x)=1-\alpha(x)$. We provide an approximation (a singular perturbation) of the semigroup generated by $A_{\mid \operatorname{ker} \Phi_{\mathrm{a}}}$ by semigroups built from those generated by $A_{\mid \operatorname{ker} \Phi_{1}}$ and $A_{\mid \operatorname{ker} \Phi_{2}}$ that supports this intuition. This result is motivated by a model of dynamics of Solea solea (Arino et al. in SIAM J Appl Math 60(2):408-436, 1999-2000; Banasiak and Goswami in Discrete Continuous Dyn Syst Ser A 35(2):617-635, 2015; Banasiak et al. in J Evol Equ 11:121-154, 2011, Mediterr J Math 11(2):533-559, 2014; Banasiak and Lachowicz in Methods of small parameter in mathematical biology, Birkhäuser, 2014; Sanchez et al. in J Math Anal Appl 323:680-699, 2006) and is, in a sense, dual to those of Bobrowski (J Evol Equ 7(3):555-565, 2007), Bobrowski and Bogucki (Stud Math 189:287-300, 2008), where semigroups generated by convex combinations of Feller's generators were studied.
\end{abstract}

\section{Introduction}

Let $L^{1}\left(\mathbb{R}^{+}\right)$be the space of Lebesgue integrable functions on $\mathbb{R}^{+}$with the norm $\|\phi\|_{L^{1}\left(\mathbb{R}^{+}\right)}=\int_{\mathbb{R}^{+}}|\phi(a)| \mathrm{d} a$, and $W^{1,1}\left(\mathbb{R}^{+}\right)$be the Sobolev space of integrable, absolutely continuous functions with derivatives in $L^{1}\left(\mathbb{R}^{+}\right)$. For nonnegative, bounded, measurable functions $b$ and $\mu$ on $\mathbb{R}^{+}$, let $A$ be the operator in $L^{1}\left(\mathbb{R}^{+}\right)$given by $A \phi=-\phi^{\prime}-\mu \phi$ with the domain composed of $\phi \in W^{1,1}\left(\mathbb{R}^{+}\right)$satisfying the boundary condition

$$
\phi(0)=\int_{\mathbb{R}^{+}} b(a) \phi(a) \mathrm{d} a .
$$

The abstract Cauchy problem related to $A$ is called the McKendrick equation [19] or Sharpe-Lotka-McKendrick equation [21,22], or Lotka-McKendrick equation [16]. In the population dynamics' interpretation of this problem, $\phi$ is an age profile of an

Mathematics Subject Classification: 47D06, 35B25, 35B40, 60J15, 34G05

Keywords: Semigroups of operators, Convergence of semigroups, Singular perturbations, Boundary conditions. 
age-structured population with births and deaths governed by $b$ and $\mu$, respectively. For this reason, it is customary to write the argument of $\phi$ as $a$, ' $a$ ' standing for 'age.'

A model of dynamics of a Solea solea or Engraulis encrasicholus population with both age and vertical structures, due to Arino et al. [1,20], uses the McKendrick model as a building block. In the model, the fish habitat is divided into $N$ spatial patches and the fish densities, or age profiles $\phi_{i}$, in the $i$ th patch satisfy the following system of equations:

$$
\begin{aligned}
\frac{\partial \phi_{i}(t, a)}{\partial t}+\frac{\partial \phi_{i}(t, a)}{\partial a} & =-\mu_{i}(a) \phi_{i}(t, a)+\epsilon^{-1} \sum_{j=1}^{N} k_{i j}(a) \phi_{j}(t, a), \\
\phi_{i}(t, 0) & =\int_{0}^{\infty} b_{i}(a) \phi_{i}(t, a) \mathrm{d} a, \quad i=1, \ldots, N,
\end{aligned}
$$

where ' $t$ ' stands for time, and $\mu_{i}$ and $b_{i}$ are age-specific and patch-specific mortality and birth rates.

In the absence of the terms $\epsilon^{-1} \sum_{j=1}^{N} k_{i j}(a) \phi_{j}(t, a)$, each patch can be treated separately and the population densities there would satisfy the McKendrick equation. The matrix $k(a)=\left(k_{i j}(a)\right)$ is composed of intensities of movements between patches that occur on a daily basis: that is, in particular, off-diagonal entries are nonnegative and the sum of entries in each column of the matrix is zero. The factor $\epsilon^{-1}$ (with $\epsilon \ll 1)$ corresponds to the fact that the age-related processes and vertical migrations (between the patches) occur at different time scales, a day being the fast time scale as compared to the fish lifetime.

The main question addressed in [1] is whether in modeling such populations one may disregard the vertical migration to work with a model that has been aggregated, or averaged, over the whole water column. To this end, the authors assume additionally that the matrix $k$ is irreducible and hence possesses the unique normalized (in the sense that $\left.v_{1}(a)+\cdots+v_{N}(a)=1\right)$ right eigenvector $\mathbf{v}(a)=\left(v_{i}(a)\right)_{i=1, \ldots, N}$, corresponding to the simple dominant eigenvalue 0 [14]. Since this vector describes the stable population distribution among the patches, e.g. [1], a heuristic argument makes plausible the ansatz that approximately we have

$$
\frac{\phi_{i}(t, a)}{\phi(t, a)}=v_{i}(a), \quad i=1, \ldots, N, a \geq 0,
$$

where $\phi=\sum_{i=1}^{N} \phi_{i}$. In other words, it is assumed that the migrations governed by $k$ occur so fast, as compared to the aging processes, that the population distribution over the patches reaches the (age-specific) equilibrium long before the aging process intervenes. This corresponds to letting $\epsilon \rightarrow 0$ in (1). In such a simplified, aggregated model, the population density satisfies the McKendrick equation with averaged birth and mortality rates:

$$
\frac{\partial \phi(t, a)}{\partial t}+\frac{\partial \phi(t, a)}{\partial a}=-\mu_{\mathrm{a}}(a) \phi(t, a)
$$




$$
\phi(t, 0)=\int_{0}^{\infty} b_{\mathrm{a}}(a) \phi(t, a) \mathrm{d} a,
$$

where 'a' stands for 'aggregated', $\mu_{\mathrm{a}}=\sum_{i=1}^{N} v_{i} \mu_{i}$ and $b_{\mathrm{a}}=\sum_{i=1}^{N} v_{i} b_{i}$. Here, the weights $v_{i}$ reflect the underlying, hidden spatial structure. Notably, since the entries of $\mathbf{v}(a)$ sum up to 1 , the limit death rate and the limit boundary condition are convex combinations of the death coefficients and the boundary conditions occurring in (1). The proofs that (3) provides an approximation to (1), and thus that the heuristic approximation (2) is justified, are given in $[1,4]$.

This effect is very similar to that observed in $[8,10,11]$ where, motivated by a number of biological models, the authors study convex combinations of Feller generators resulting from 'averaging' the stochastic processes involved. In fact, these two effects are in a sense dual: Under certain regularity conditions on the model's parameters, the predual of the McKendrick semigroup may be constructed in a space of continuous functions [10]. Then, a perturbation of a boundary condition becomes a perturbation of the generator, and the convergence discussed above may be put in the context of $[8,10,11]$, see $[10]$ for details.

In [3-6], the problem of the convergence of solutions to (1) as $\epsilon \rightarrow 0$ was fully solved using asymptotic analysis (even in a more general model). However, the authors did not consider the problem as an example of a convex combination of boundary conditions, and their reasonings are based on special properties of the McKendrick semigroup. In this paper, we put the problem in the framework of Greiner [15] to deal with abstract operators and abstract boundary conditions and we approximate the semigroup with the generator's domain equal to $\operatorname{ker}\left[\Phi_{1} \circ \alpha+\Phi_{2} \circ(1-\alpha)\right]$ by a family of semigroups with the generators' domains involving $\operatorname{ker} \Phi_{1}$ and $\operatorname{ker} \Phi_{2}$ - see Theorems 2.1 and 2.2. This approximation supports the intuition that the former semigroup is in a sense an average of the latter semigroups. Note that the problem posed here is in a sense converse to the result of $[1,3]$ : There, a complex model is reduced to a simpler one involving convex combination of the boundary conditions while here, given the semigroup generated by an operator related to a convex combination of boundary conditions, we construct an approximating sequence of semigroups with desired averaging properties.

\section{The main theorem}

For our main result, we adopt the following abstract approach to boundary conditions, due to Greiner [15]. Let $\mathbb{X}$ and $\mathbb{Y}$ be two Banach spaces, $A: D(A) \rightarrow \mathbb{X}$ be a closed operator in $\mathbb{X}$, and $L: D(A) \rightarrow \mathbb{Y}$ be a linear operator which is continuous with respect to the graph norm in $D(A)$. Moreover, assume $L$ to be surjective, and suppose that $A_{0}$, defined as the restriction of $A$ to ker $L$, generates a semigroup of operators in $\mathbb{X}$. The main question studied in [15] was whether, given $F \in \mathcal{L}(\mathbb{X}, \mathbb{Y})$, the operator $A_{F}$ defined as the restriction of $A$ to $\operatorname{ker}(L-F)$ is the generator as well. While in general (see [15, Example 1.5]), the answer is in the negative, Greiner's first fundamental theorem [15, Thm 2.1] establishes that $A_{F}$ is the generator for any $F$ 
provided there is a constant $\gamma$ such that for $\lambda$ larger than some $\lambda_{0}$

$$
\|L x\| \geq \lambda \gamma\|x\|, \quad \text { for all } x \in \operatorname{ker}(\lambda-A) .
$$

Throughout the paper, we will assume that (5) holds.

Note that the boundary conditions in the model (1) fit into this abstract framework, with $L$ being the trace operator at $a=0$ and $F$ the bounded integral operator on the right-hand side of the boundary conditions-see Example 1 for details. Moreover, following the structure of the limit problem (4), we consider an abstract convex combination of two boundary value problems by introducing two boundary operators $F_{1}, F_{2} \in \mathcal{L}(\mathbb{X}, \mathbb{Y})$ and a bounded linear operator $\alpha \in \mathcal{L}(\mathbb{X})$ and define

$$
F_{\mathrm{a}}=F_{1} \alpha+F_{2} \beta,
$$

where $\beta=I_{\mathbb{X}}-\alpha$ ('a' for 'average'). By Greiner's theorem, $A_{i}:=A_{F_{i}}$ and $A_{\mathrm{a}}:=A_{F_{\mathrm{a}}}$ are generators with $D\left(A_{i}\right)=\operatorname{ker} \Phi_{i}$, where $\Phi_{i}=L-F_{i}, i=1,2$ and $D\left(A_{\mathrm{a}}\right)=$ $\operatorname{ker} \Phi_{\mathrm{a}}$, where $\Phi_{\mathrm{a}}=L-F_{\mathrm{a}}$. Observe that in this formulation, there is no need to refer anymore to the vector $\mathbf{v}$, whose role has been taken over by the operators $\alpha$ and $\beta$ satisfying $\alpha+\beta=I_{\mathbb{X}}$.

Our main goal is to approximate $\left(\mathrm{e}^{t A_{\mathrm{a}}}\right)_{t \geq 0}$ by means of semigroups built from $\left(\mathrm{e}^{t A_{1}}\right)_{t \geq 0}$ and $\left(\mathrm{e}^{t A_{2}}\right)_{t \geq 0}$. To this end, we introduce operators $\mathcal{A}_{\kappa}, \kappa>0$, in $\mathbb{X} \times \mathbb{X}$ given by

$$
\begin{aligned}
D\left(\mathcal{A}_{\kappa}\right) & =D\left(A_{1}\right) \times D\left(A_{2}\right)=\operatorname{ker} \Phi_{1} \times \operatorname{ker} \Phi_{2}, \\
\mathcal{A}_{\kappa} & =\left(\begin{array}{cc}
A_{1} & 0 \\
0 & A_{2}
\end{array}\right)+\kappa\left(\begin{array}{cc}
-\beta & \alpha \\
\beta & -\alpha
\end{array}\right)=: \mathcal{A}_{0}+\kappa \mathcal{Q} .
\end{aligned}
$$

We assume that the semigroup generated by $\mathcal{A}_{0}$, say $\left(\mathrm{e}^{t \mathcal{A}_{0}}\right)_{t \geq 0}$ is semicontractive, i.e., it satisfies

$$
\left\|\mathrm{e}^{t \mathcal{A}_{0}}\right\| \leq \mathrm{e}^{\omega t}, \quad t \geq 0
$$

for some $\omega \in \mathbb{R}$ and that

$$
\mathcal{P}:=\mathcal{Q}+I_{\mathbb{X} \times \mathbb{X}}=\left(\begin{array}{ll}
\alpha & \alpha \\
\beta & \beta
\end{array}\right)
$$

is a contraction in $\mathbb{X} \times \mathbb{X}$. (The former condition is automatically satisfied if $\left(\mathrm{e}^{t A_{0}}\right)_{t \geq 0}$ is semicontractive - see the remark on page 215 in [15].) We note that $\mathcal{P}$ is idempotent, hence

$$
\begin{aligned}
\mathrm{e}^{\kappa t \mathcal{Q}} & =\mathrm{e}^{-\kappa t} \mathrm{e}^{\kappa t \mathcal{P}}=\mathrm{e}^{-\kappa t}\left[I_{\mathbb{X} \times \mathbb{X}}+\left(\mathrm{e}^{t \kappa}-1\right) \mathcal{P}\right] \\
& =\mathrm{e}^{-\kappa t} I_{\mathbb{X} \times \mathbb{X}}+\left(1-\mathrm{e}^{-\kappa t}\right) \mathcal{P} .
\end{aligned}
$$

It follows that $\left\|\mathrm{e}^{t \mathcal{Q}}\right\| \leq 1$ and for the semigroups generated by $\mathcal{A}_{\kappa}$ (which exist by the Phillips perturbation theorem), we have, by the Trotter product formula,

$$
\left\|\mathrm{e}^{t \mathcal{A}_{\kappa}} x\right\| \leq \lim _{n \rightarrow \infty}\left\|\left[\mathrm{e}^{\frac{t}{n} \mathcal{A}_{0}} \mathrm{e}^{\frac{\kappa t}{n} \mathcal{Q}}\right]^{n} x\right\| \leq \mathrm{e}^{\omega t}\|x\|, \quad x \in \mathbb{X} \times \mathbb{X},
$$


so that

$$
\left\|\mathrm{e}^{t \mathcal{A}_{\kappa}}\right\| \leq \mathrm{e}^{\omega t}, \quad \kappa>0, t \geq 0 .
$$

Operator $\mathcal{P}$ is a projection on the subspace $\mathbb{X}^{\prime} \subset \mathbb{X} \times \mathbb{X}$ of vectors of the form $\left(\begin{array}{l}\alpha x \\ \beta x\end{array}\right)$; the latter space is isomorphic to $\mathbb{X}$ with isomorphism $\mathcal{I}: \mathbb{X} \rightarrow \mathbb{X}^{\prime}$ given by $\mathcal{I} x=\left(\begin{array}{l}\alpha x \\ \beta x\end{array}\right)$.

THEOREM 2.1. In the above setup, assume that $\alpha$ leaves $D(A)$ invariant. Then,

$$
\lim _{\kappa \rightarrow+\infty} \mathrm{e}^{t \mathcal{A}_{\kappa}}\left(\begin{array}{l}
x_{1} \\
x_{2}
\end{array}\right)=\mathcal{I} \mathrm{e}^{t A_{\mathrm{a}}} \mathcal{I}^{-1} \mathcal{P}\left(\begin{array}{l}
x_{1} \\
x_{2}
\end{array}\right)=\left(\begin{array}{c}
\alpha \mathrm{e}^{t \mathcal{A}_{\mathrm{a}}}\left(x_{1}+x_{2}\right) \\
\beta \mathrm{e}^{t \mathcal{A}_{\mathrm{a}}\left(x_{1}+x_{2}\right)}
\end{array}\right), \quad t>0, x_{1}, x_{2} \in \mathbb{X} .
$$

For $\left(\begin{array}{l}x_{1} \\ x_{2}\end{array}\right) \in \mathbb{X}^{\prime}$, the same is true for $t=0$ as well and the limit is almost uniform in $t \in[0, \infty)$; for other $\left(\begin{array}{l}x_{1} \\ x_{2}\end{array}\right)$, the limit is almost uniform in $t \in(0, \infty)$.

Intuitively, this result may be explained as follows. The components of the semigroup $\left(\mathrm{e}^{t \mathcal{A}_{0}}\right)_{t \geq 0}$ are uncoupled, while in $\left(\mathrm{e}^{t \mathcal{A}_{\kappa}}\right)_{t \geq 0}$, the coupling is realized by the operator $\mathcal{Q}$ which may be thought of as describing a Markov chain switching one dynamics into the other (the jumps' intensities are state-dependent, see examples given later). As $\kappa \rightarrow \infty$, the Markov chain reaches its statistical equilibrium, so that with 'probability' $\alpha$, it chooses the first dynamics, and with 'probability' $\beta$, it chooses the second dynamics. This results in a convex combination of boundary conditions in the limit semigroup. (Compare the main theorem in [8], see also [10].)

THEOREM 2.2. Under conditions of the previous theorem, let $\mathcal{B}=\left(\begin{array}{cc}B_{1} & 0 \\ 0 & B_{2}\end{array}\right)$, where $B_{1}$ and $B_{2}$ are bounded linear operators. Then,

$$
\lim _{\kappa \rightarrow+\infty} \mathrm{e}^{t\left(\mathcal{A}_{\kappa}+\mathcal{B}\right)}\left(\begin{array}{l}
x_{1} \\
x_{2}
\end{array}\right)=\mathcal{I} \mathrm{e}^{t\left(A_{\mathrm{a}}+B_{1} \alpha+B_{2} \beta\right)} \mathcal{I}^{-1} \mathcal{P}\left(\begin{array}{l}
x_{1} \\
x_{2}
\end{array}\right), \quad t>0, x_{1}, x_{2} \in \mathbb{X} .
$$

For $\left(\begin{array}{l}x_{1} \\ x_{2}\end{array}\right) \in \mathbb{X}^{\prime}$, the same is true for $t=0$ as well and the limit is almost uniform in $t \in[0, \infty)$; for other $\left(\begin{array}{l}x_{1} \\ x_{2}\end{array}\right)$, the limit is almost uniform in $t \in(0, \infty)$.

We will prove these theorems in Sect. 5; in Sect. 3, we give examples of applications, and in Sect. 4, we provide preparatory lemmas.

REMARK 1. For Theorems 2.1 and 2.2, besides (5) and (6), we assume that $\mathcal{P}$, defined in (7), is a contraction in $\mathbb{X} \times \mathbb{X}$ and $\alpha$ leaves $D(A)$ invariant. While the nature of the first and the last conditions is transparent, the other two require a comment. As already mentioned, together they imply stability condition (9) (which is a common assumption in convergence theorems), and in fact, our theorems remain true if we simply assume (9). However, for the sake of applications, it is more convenient to assume the two conditions discussed above. Out of these two, the one requiring $\mathcal{P}$ to be a contraction seems to be most restrictive, apparently excluding spaces with supremum norm. On the other hand, this assumption is often satisfied in $L^{1}$-type spaces. (Similarly, the 'dual' theorem in $[8,10]$ is designed for spaces of continuous functions.) In particular, if $\mathbb{X}$ is an AL-space, i.e., a Banach lattice such that

$$
\|x+y\|=\|x\|+\|y\|, \quad x, y \geq 0,
$$


and $\mathbb{X} \times \mathbb{X}$ is equipped with the order ' $\left(\begin{array}{l}x \\ y\end{array}\right) \geq 0$ iff $x \geq 0$ and $y \geq 0$ ' and the norm $\left\|\left(\begin{array}{l}x \\ y\end{array}\right)\right\|=\|x\|+\|y\|$, then $\mathcal{P}$ is a contraction provided $\alpha$ and $\beta$ are positive operators. For, in such a case,

$$
\begin{aligned}
\left\|\mathcal{P}\left(\begin{array}{l}
x \\
y
\end{array}\right)\right\| & =\left\|\left(\begin{array}{l}
\alpha(x+y) \\
\beta(x+y)
\end{array}\right)\right\|=\|\alpha(x+y)\|+\|\beta(x+y)\|=\|x+y\| \\
& \leq\left\|\left(\begin{array}{l}
x \\
y
\end{array}\right)\right\|, \quad x, y \geq 0,
\end{aligned}
$$

and $\mathcal{P}$ is positive. Hence (see e.g. [2, Proposition 2.67]),

$$
\|\mathcal{P}\|=\sup _{\left\|\left(\begin{array}{l}
x \\
y
\end{array}\right)\right\|=1,\left(\begin{array}{l}
x \\
y
\end{array}\right) \geq 0}\left\|\mathcal{P}\left(\begin{array}{l}
x \\
y
\end{array}\right)\right\| \leq 1 .
$$

\section{Examples}

EXAMPLE 1. In the motivating example of the fish population dynamics,

$$
A: W^{1,1}\left(\mathbb{R}^{+}\right) \rightarrow L^{1}\left(\mathbb{R}^{+}\right) \quad A \phi=-\phi^{\prime},
$$

and $L: W^{1,1}\left(\mathbb{R}^{+}\right) \rightarrow \mathbb{R}$ is given by $L \phi=\phi(0)$. Here, $\operatorname{ker}(\lambda-A), \lambda>0$ is spanned by $e_{\lambda}$ where $e_{\lambda}(a)=\mathrm{e}^{-\lambda a}, a \geq 0$. Since $L e_{\lambda}=1$ and $\left\|e_{\lambda}\right\|=\frac{1}{\lambda}$, condition (5) is satisfied with $\gamma=1$ (in fact, we have equality there).

For $b_{i} \in L^{\infty}\left(\mathbb{R}^{+}\right), i=1,2$, the functionals $F_{i} \phi=\int_{0}^{\infty} b_{i}(a) \phi(a) \mathrm{d} a$ are linear and bounded. Hence, $A_{F_{i}}$ generates a semigroup of operators and so does $A_{F_{i}}+B_{i}$, where given $\mu_{i} \in L^{\infty}\left(\mathbb{R}^{+}\right), B_{i}$ is a (bounded) multiplication operator $\phi \mapsto-\mu_{i} \phi$. It is well known (see e.g. $[9,12,16]$ ) that there is $\omega$ such that $\left\|\mathrm{e}^{t\left(A_{F_{i}}+B_{i}\right)}\right\| \leq \mathrm{e}^{\omega t}, i=1,2$, implying (6).

Let $\alpha \in W^{1, \infty}\left(\mathbb{R}^{+}\right)$satisfy $0 \leq \alpha \leq 1$. Then the related multiplication operator (denoted in what follows by the same letter) is bounded in $L^{1}\left(\mathbb{R}^{+}\right)$and leaves $D(A)=$ $W^{1,1}\left(\mathbb{R}^{+}\right)$invariant. Moreover, the related operator $\mathcal{P}[$ see $(7)]$ in $L^{1}\left(\mathbb{R}^{+}\right) \times L^{1}\left(\mathbb{R}^{+}\right)$, equipped with the norm $\left\|\left(\begin{array}{l}\phi_{1} \\ \phi_{2}\end{array}\right)\right\|=\left\|\phi_{1}\right\|_{L^{1}\left(\mathbb{R}^{+}\right)}+\left\|\phi_{2}\right\|_{L^{1}\left(\mathbb{R}^{+}\right)}$, is a contraction (see Remark 1). Hence, all assumptions of Theorem 2.2 are satisfied. This again establishes that the general model (1) (with $N=2$ and normalized matrix $k$ ) may be approximated by the averaged one (3).

EXAMPLE 2. Let $\mathbb{X}=L^{1}(\mathbb{R})$. For our purposes, it will be convenient to identify $\phi \in L^{1}(\mathbb{R})$ with the pair $\left(\phi_{j}\right)_{j \in \mathbb{J}}$ of functions on $\mathbb{R}^{+}$defined by $\phi_{j}(a)=\phi(j a), a \geq 0$, where $\mathbb{J}=\{-1,1\}$. Certainly $\phi_{j} \in L^{1}\left(\mathbb{R}^{+}\right)$, i.e., we identify $L^{1}(\mathbb{R})$ with $L^{1}\left(\mathbb{R}^{+}\right) \times$ $L^{1}\left(\mathbb{R}^{+}\right)$. With this identification in mind, and given constants $k_{j} \geq 0, \sigma_{j}>0, j \in \mathbb{J}$, we define an operator $A$ in $L^{1}\left(\mathbb{R}^{+}\right)$by

$$
A\left(\phi_{j}\right)_{j \in \mathbb{J}}=\left(\sigma_{j}^{2} \phi_{j}^{\prime \prime}\right)_{j \in \mathbb{J}}, \quad D(A)=W^{2,1}\left(\mathbb{R}^{+}\right) \times W^{2,1}\left(\mathbb{R}^{+}\right),
$$


and $L: D(A) \rightarrow \mathbb{R}^{2}$, by

$$
L\left(\phi_{j}\right)_{j \in \mathbb{J}}=\left(\phi_{j}^{\prime}(0)-k_{j} \phi_{j}(0)\right)_{j \in \mathbb{J}} .
$$

The semigroup generated by $A_{0}$, the restriction of $A$ to ker $L$, is composed of Markov operators in $L^{1}(\mathbb{R})$ and describes dynamics of distributions of two independent elastic Brownian motions on two half-axes, with two different elasticity coefficients $\left(k_{j}\right)$ in each half-axis, and no communication between the half-axes.

The subspace $\operatorname{ker}(\lambda-A)$ is composed of vectors of the form $\left(\phi_{j}\right)_{j \in \mathbb{J}}=\left(C_{j} e_{\lambda_{j}}\right)_{j \in \mathbb{J}}$, where $C_{j}$ are arbitrary constants, $\lambda_{j}=\frac{\sqrt{\lambda}}{\sigma_{j}}$, and $e_{\lambda}$ was defined in the previous example. For such vectors and $L^{1}$-type norm in $\mathbb{R}^{2}$,

$$
\begin{aligned}
\left\|L\left(\phi_{j}\right)_{j \in \mathbb{J}}\right\| & =\sum_{j \in \mathbb{J}}\left|\phi_{j}^{\prime}(0)-k_{j} \phi_{j}(0)\right|=\sum_{j \in \mathbb{J}}\left(\frac{\sqrt{\lambda}}{\sigma_{i}}+k_{j}\right)\left|C_{j}\right| \geq \frac{\sqrt{\lambda}}{\sigma} \sum_{j \in \mathbb{J}}\left|C_{j}\right|, \\
\lambda\left\|\left(\phi_{j}\right)_{j \in \mathbb{J}}\right\| & =\sqrt{\lambda} \sum_{j \in \mathbb{J}} \sigma_{j}\left|C_{j}\right| \leq \sigma \sqrt{\lambda} \sum_{j \in \mathbb{J}}\left|C_{j}\right|, \quad \lambda>0,
\end{aligned}
$$

where $\sigma=\max \left\{\sigma_{1}, \sigma_{-1}\right\}$. Hence, condition (5) is satisfied with $\gamma=\sigma^{-2}$.

In accordance with the theory described in Sect. 2, we define the boundary operators $F_{1}$ and $F_{2}$ from $\mathbb{X}$ to $\mathbb{Y}=\mathbb{R}^{2}$ by

$$
\phi \rightarrow\left(\int_{\mathbb{R}} f_{j, i} \phi\right)_{j \in \mathbb{J}}, \quad i=1,2,
$$

where $f_{j, i} \in L^{\infty}(\mathbb{R}), j \in \mathbb{J}, i=1,2$ are given functions. Then $A$ restricted to $\left(\phi_{j}\right)_{j \in \mathbb{J}}=\phi \in L^{1}(\mathbb{R})$ satisfying

$$
\phi_{j}^{\prime}(0)-k_{j} \phi_{j}(0)=\int_{\mathbb{R}} f_{j, i} \phi, \quad j \in \mathbb{J},
$$

is the generator of a semicontractive semigroup in $L^{1}(\mathbb{R})$ for both $i$, and (6) is satisfied. The boundary conditions (13) are a version of nonlocal Robin boundary conditions which may model two populations occupying (one-dimensional) domains with joint boundary, in which the flux of individuals across the common boundary is proportional not only to the difference of the densities at the boundary but also to the (weighted) difference of total numbers of individuals in each domain.

For sufficiently regular $0 \leq \alpha \leq 1$, the related multiplication operator in $L^{1}(\mathbb{R})$ leaves $D(A)$ invariant, and the related operator $\mathcal{P}$ is a contraction (see Remark 1). Therefore Theorem 2.1 ensures that in the limit (10), we obtain (an isomorphic copy) of the semigroup generated by $A$ restricted to $\left(\phi_{j}\right)_{j \in \mathbb{J}}=\phi \in L^{1}(\mathbb{R})$ which satisfy

$$
\phi_{j}^{\prime}(0)-k_{j} \phi_{j}(0)=\int_{\mathbb{R}}\left(\alpha f_{j, 1}+\beta f_{j, 2}\right) \phi, \quad j \in \mathbb{J} ;
$$

the right-hand side here is a convex combination of the right-hand sides in (13). 


\section{Auxiliary results}

In this section, we present preparatory results for the proofs of the main theorems, to be given in the following section.

We begin by recalling the basic structure related to assumption (5) (see [15, Lemma 1.2]). We fix $\lambda>\lambda_{0}$ in the resolvent set of $A_{0}$, and consider an $x \in D(A)$. Since $A_{0}$ is a generator, we can choose $x_{1} \in D\left(A_{0}\right)$ so that $\left(\lambda-A_{0}\right) x_{1}=(\lambda-A) x$. Writing $x_{2}=x-x_{1}$, we have

$$
x=x_{1}+x_{2}
$$

with $x_{1} \in D\left(A_{0}\right)$ and $x_{2} \in \operatorname{ker}(\lambda-A)$. Such a decomposition of $x$ is unique, because otherwise we could find a nonzero $y \in D\left(A_{0}\right) \cap \operatorname{ker}(\lambda-A)$, implying that $y$ is an eigenvector of $A_{0}$ related to $\lambda$, a contradiction.

Since $L$ is assumed to be surjective, condition (5) shows, by the open mapping theorem, that $L$ restricted to $\operatorname{ker}(\lambda-A)$ is an isomorphism of $\operatorname{ker}(\lambda-A)$ (with the graph norm) and $\mathbb{Y}$. Following Greiner, by $L_{\lambda}$, we denote the inverse of $L_{\mid \operatorname{ker}(\lambda-A)}$.

LEMMA 4.1. Let $\mathfrak{A}_{\kappa}$ denote the extension of $\mathcal{A}_{\kappa}$ to the maximal domain $D(A) \times$ $D(A)$ and let $\Phi: \mathbb{X} \times \mathbb{X} \rightarrow \mathbb{Y} \times \mathbb{Y}$ be given by $\Phi\left(\begin{array}{l}x_{1} \\ x_{2}\end{array}\right)=\left(\begin{array}{l}\Phi_{1} x_{1} \\ \Phi_{2} x_{2}\end{array}\right)$. For $x \in D\left(A_{a}\right)$, we define

$$
\begin{aligned}
& x_{1, \kappa}=\alpha x+L_{\kappa} \tilde{x}, \\
& x_{2, \kappa}=\beta x-L_{\kappa} \tilde{x},
\end{aligned}
$$

where $\tilde{x}:=F_{1} \alpha x-L \alpha x=L \beta x-F_{2} \beta x \in \mathbb{Y}$, by $D\left(A_{a}\right)=\operatorname{ker} \Phi_{a}$. Then,

$$
\mathfrak{A}_{\kappa}\left(\begin{array}{l}
x_{1, \kappa} \\
x_{2, \kappa}
\end{array}\right)=\left(\begin{array}{l}
A \alpha x \\
A \beta x
\end{array}\right), \lim _{\kappa \rightarrow+\infty}\left(\begin{array}{l}
x_{1, \kappa} \\
x_{2, \kappa}
\end{array}\right)=\left(\begin{array}{c}
\alpha x \\
\beta x
\end{array}\right) \quad \text { and } \lim _{\kappa \rightarrow+\infty} \Phi\left(\begin{array}{l}
x_{1, \kappa} \\
x_{2, \kappa}
\end{array}\right)=0 \text {. }
$$

Proof. Using $A L_{\kappa} \tilde{x}=\kappa L_{\kappa} \tilde{x}$, we get

$\mathcal{A}_{0}\left(\begin{array}{l}x_{1, \kappa} \\ x_{2, \kappa}\end{array}\right)=\left(\begin{array}{c}A \alpha x+\kappa L_{\kappa} \tilde{x} \\ A \beta x-\kappa L_{\kappa} \tilde{x}\end{array}\right) \quad$ while $\quad \mathcal{Q}\left(\begin{array}{c}x_{1, \kappa} \\ x_{2, \kappa}\end{array}\right)=\left(\begin{array}{c}-\beta L_{\kappa} \tilde{x}-\alpha L_{\kappa} \tilde{x} \\ \beta L_{\kappa} \tilde{x}+\alpha L_{\kappa} \tilde{x}\end{array}\right)=\left(\begin{array}{c}-L_{\kappa} \tilde{x} \\ L_{\kappa} \tilde{x}\end{array}\right) ;$

this shows the first part. Next, by (5), we have $\left\|L_{\kappa} \tilde{x}\right\| \leq \frac{1}{\kappa \gamma}\|\tilde{x}\|$. It follows that $\lim _{\kappa \rightarrow+\infty}\left(\begin{array}{l}x_{1, \kappa} \\ x_{2, \kappa}\end{array}\right)=\left(\begin{array}{l}\alpha x \\ \beta x\end{array}\right)$ and

$$
\Phi\left(\begin{array}{l}
x_{1, \kappa} \\
x_{2, \kappa}
\end{array}\right)=\left(\begin{array}{l}
L \alpha x+\tilde{x}-F_{1} \alpha x-F_{1} L_{\kappa} \tilde{x} \\
L \beta x-\tilde{x}+F_{2} \beta x+F_{2} L_{\kappa} \tilde{x}
\end{array}\right) \underset{\kappa \rightarrow \infty}{\longrightarrow}\left(\begin{array}{l}
L \alpha x+\tilde{x}-F_{1} \alpha x \\
L \beta x-\tilde{x}+F_{2} \beta x
\end{array}\right)=\left(\begin{array}{l}
0 \\
0
\end{array}\right) .
$$

This completes the proof.

Unfortunately, in general, $\left(\begin{array}{l}x_{1, \kappa} \\ x_{2, \kappa}\end{array}\right)$ does not belong to $D\left(\mathcal{A}_{\kappa}\right)$. Our main goal is to modify this vector appropriately without altering its crucial properties (see Lemma 4.4). To this end, we need the following two lemmas. 
LEMMA 4.2. Let $\lambda>\max \left(\lambda_{0}, \omega\right)$. Then $\operatorname{ker}\left(\lambda-\mathfrak{A}_{\kappa}\right)$ is composed of vectors of the form

$$
\left(\begin{array}{l}
x_{1} \\
x_{2}
\end{array}\right)=\left(\begin{array}{c}
\kappa\left(\lambda+\kappa-A_{0}\right)^{-1} \alpha h_{\lambda}-h_{\lambda+\kappa} \\
h_{\lambda}+h_{\lambda+\kappa}-\kappa\left(\lambda+\kappa-A_{0}\right)^{-1} \alpha h_{\lambda}
\end{array}\right),
$$

where $h_{\lambda} \in \operatorname{ker}(\lambda-A)$ and $h_{\lambda+\kappa} \in \operatorname{ker}(\lambda+\kappa-A)$ may be chosen arbitrarily.

Proof. The operator $\mathcal{I}=\left(\begin{array}{cc}\alpha & -I_{\mathbb{X}} \\ \beta & I_{\mathbb{X}}\end{array}\right)$ is an isomorphism of $\mathbb{X} \times \mathbb{X}$ with the inverse $\left(\begin{array}{cc}I_{\mathbb{X}} & I_{\mathbb{X}} \\ -\beta & \alpha\end{array}\right)$. A vector $x=\left(\begin{array}{l}x_{1} \\ x_{2}\end{array}\right)$ satisfies $\lambda x-\mathfrak{A}_{\kappa} x=0$ iff for $y=\mathcal{I}^{-1} x$ we have

$$
\lambda y-\mathcal{I}^{-1} \mathfrak{A}_{\kappa} \mathcal{I} y=0 .
$$

On the other hand,

$$
\mathcal{I}^{-1} \mathfrak{A}_{\kappa} \mathcal{I}=\left(\begin{array}{cc}
A \alpha+A \beta & 0 \\
-\beta A \alpha+\alpha A \beta & \beta A+\alpha A-\kappa
\end{array}\right)=\left(\begin{array}{cc}
A & 0 \\
\alpha A-A \alpha & A-\kappa
\end{array}\right) .
$$

Hence, (18) is satisfied for $y=\left(\begin{array}{l}y_{1} \\ y_{2}\end{array}\right)$ iff

$$
\lambda y_{1}-A y_{1}=0 \quad \text { and } \quad(\lambda+\kappa) y_{2}-A y_{2}=(\alpha A-A \alpha) y_{1} .
$$

The first condition here means that $y_{1}$ is a member, say $h_{\lambda}$, of $\operatorname{ker}(\lambda-A)$. Consequently, the other condition may be written in the form

$$
(\lambda+\kappa) y_{2}-A y_{2}=(\lambda+\kappa-A) \alpha h_{\lambda}-\kappa \alpha h_{\lambda}
$$

or $(\lambda+\kappa-A)\left(y_{2}-\alpha h_{\lambda}\right)=-\kappa \alpha h_{\lambda}$. Therefore, by (14), $y_{2}-\alpha h_{\lambda}$ differs from $-\kappa\left(\lambda+\kappa-A_{0}\right)^{-1} \alpha h_{\lambda}$ by an element of $\operatorname{ker}(\lambda+\kappa-A)$, say $h_{\lambda+\kappa}$. This is equivalent to saying that

$$
y_{2}=\alpha h_{\lambda}+h_{\lambda+\kappa}-\kappa\left(\lambda+\kappa-A_{0}\right)^{-1} \alpha h_{\lambda} .
$$

To complete the proof, it suffices to calculate $\left(\begin{array}{l}x_{1} \\ x_{2}\end{array}\right)=\mathcal{I}\left(\begin{array}{l}y_{1} \\ y_{2}\end{array}\right)$.

LEMMA 4.3. There is $\lambda_{1}$ such that for $\lambda>\lambda_{1}$ and all $\kappa>0$

$$
\left\|\Phi_{1} x_{1}\right\|+\left\|\Phi_{2} x_{2}\right\| \geq\left\|x_{1}\right\|+\left\|x_{2}\right\| \quad \text { for all }\left(\begin{array}{l}
x_{1} \\
x_{2}
\end{array}\right) \in \operatorname{ker}\left(\lambda-\mathfrak{A}_{\kappa}\right) .
$$

Proof. Fix $\lambda>\max \left(\lambda_{0}, \omega\right)$ and $\kappa>0$. By Lemma 4.2, a vector $\left(\begin{array}{l}x_{1} \\ x_{2}\end{array}\right) \in \operatorname{ker}\left(\lambda-\mathfrak{A}_{\kappa}\right)$ is of the form (17). Using (6) and then (5),

$$
\begin{aligned}
\left\|x_{1}\right\|+\left\|x_{2}\right\| & \leq \frac{2 \kappa\|\alpha\|}{\lambda \gamma(\lambda+\kappa-\omega)}\left\|L h_{\lambda}\right\|+\frac{2}{\gamma(\lambda+\kappa)}\left\|L h_{\lambda+\kappa}\right\|+\frac{1}{\gamma \lambda}\left\|L h_{\lambda}\right\| \\
& \leq \frac{K}{\lambda}\left(\left\|L h_{\lambda}\right\|+\left\|L h_{\lambda+\kappa}\right\|\right)
\end{aligned}
$$


for an appropriately chosen constant $K>0$. Since $F_{1}$ and $F_{2}$ are bounded operators,

$$
\left\|F_{1} x_{1}\right\|+\left\|F_{2} x_{2}\right\| \leq \frac{K}{\lambda}\left(\left\|L h_{\lambda}\right\|+\left\|L h_{\lambda+\kappa}\right\|\right)
$$

with possibly different $K$.

On the other hand, the map $\mathbb{Y} \times \mathbb{Y} \ni\left(y_{1}, y_{2}\right) \mapsto\left\|y_{2}\right\|+\left\|y_{1}+y_{2}\right\|$ is a norm in $\mathbb{Y} \times \mathbb{Y}$ and $\left\|y_{2}\right\|+\left\|y_{1}+y_{2}\right\| \leq 2\left(\left\|y_{1}\right\|+\left\|y_{2}\right\|\right)$. Hence, by the Open Mapping Theorem, $\left\|y_{1}\right\|+\left\|y_{2}\right\| \leq \mu\left(\left\|y_{2}\right\|+\left\|y_{1}+y_{2}\right\|\right)$ for some constant $\mu>0$ and all $y_{1}, y_{2} \in \mathbb{Y}$. It follows that $\left(\right.$ recall $\left.\left(\lambda+\kappa-A_{0}\right)^{-1} \alpha h_{\lambda} \in D\left(A_{0}\right)=\operatorname{ker} L\right)$,

$$
\begin{aligned}
\left\|\Phi_{1} x_{1}\right\|+\left\|\Phi_{2} x_{2}\right\| & =\left\|L h_{\lambda+\kappa}+F_{1} x_{1}\right\|+\left\|L h_{\lambda}+L h_{\lambda+\kappa}-F_{2} x_{2}\right\| \\
& \geq\left\|L h_{\lambda+\kappa}\right\|+\left\|L h_{\lambda}+L h_{\lambda+\kappa}\right\|-\left\|F_{1} x_{1}\right\|-\left\|F_{2} x_{2}\right\| \\
& \geq\left(\frac{1}{\mu}-\frac{K}{\lambda}\right)\left(\left\|L h_{\lambda}\right\|+\left\|L h_{\lambda+\kappa}\right\|\right) \\
& \geq \frac{K}{\lambda}\left(\left\|L h_{\lambda}\right\|+\left\|L h_{\lambda+\kappa}\right\|\right) \\
& \geq\left\|x_{1}\right\|+\left\|x_{2}\right\|,
\end{aligned}
$$

provided $\lambda \geq 2 K \mu$.

The map $\Phi$ from Lemma 4.1 is surjective. Arguing as in the proof of the decomposition (14), we see that any vector $v \in \mathbb{X} \times \mathbb{X}$ may be uniquely written as $v=v_{1}+v_{2}$ where $v_{1} \in \operatorname{ker} \Phi=D\left(\mathcal{A}_{\kappa}\right)$, and $v_{2} \in \operatorname{ker}\left(\lambda-\mathfrak{A}_{\kappa}\right)$, where $\lambda>\max \left(\lambda_{1}, \omega\right)$ and $\kappa>0$ are fixed $\left(\lambda_{1}\right.$ is defined in Lemma 4.3). Hence, $\Phi$ restricted to $\operatorname{ker}\left(\lambda-\mathfrak{A}_{\kappa}\right)$ is surjective as well, and inequality (19) shows that it is invertible, with inverse, say $J_{\lambda, \kappa}$, of norm at most 1 .

LEMMA 4.4. For $x \in D\left(A_{a}\right)$, there are $\left(\begin{array}{l}y_{1, \kappa} \\ y_{2, \kappa}\end{array}\right) \in D\left(A_{1}\right) \times D\left(A_{2}\right)=D\left(\mathcal{A}_{\kappa}\right)$ such that

$$
\lim _{\kappa \rightarrow+\infty}\left(\begin{array}{l}
y_{1, \kappa} \\
y_{2, \kappa}
\end{array}\right)=\left(\begin{array}{c}
\alpha x \\
\beta x
\end{array}\right) \quad \text { and } \quad \lim _{\kappa \rightarrow+\infty} \mathcal{A}_{\kappa}\left(\begin{array}{l}
y_{1, \kappa} \\
y_{2, \kappa}
\end{array}\right)=\left(\begin{array}{c}
A \alpha x \\
A \beta x
\end{array}\right) \text {. }
$$

Proof. Take $\lambda>\lambda_{1}$ and define (see Lemma 4.1)

$$
\left(\begin{array}{l}
y_{1, \kappa} \\
y_{2, \kappa}
\end{array}\right):=\left(\begin{array}{l}
x_{1, \kappa} \\
x_{2, \kappa}
\end{array}\right)-J_{\lambda, \kappa} \Phi\left(\begin{array}{l}
x_{1, \kappa} \\
x_{2, \kappa}
\end{array}\right)
$$

Then, $\Phi\left(\begin{array}{l}y_{1, \kappa} \\ y_{2, \kappa}\end{array}\right)=\Phi\left(\begin{array}{l}x_{1, \kappa} \\ x_{2, \kappa}\end{array}\right)-\Phi\left(\begin{array}{l}x_{1, \kappa} \\ x_{2, \kappa}\end{array}\right)=0$, proving that $\left(\begin{array}{l}y_{1, \kappa} \\ y_{2, \kappa}\end{array}\right) \in D\left(\mathcal{A}_{\kappa}\right)$. Also, by Lemma 4.1 ,

$$
\lim _{\kappa \rightarrow+\infty}\left(\begin{array}{l}
y_{1, \kappa} \\
y_{2, \kappa}
\end{array}\right)=\left(\begin{array}{c}
\alpha x \\
\beta x
\end{array}\right)-\lim _{\kappa \rightarrow+\infty} J_{\lambda, \kappa} \Phi\left(\begin{array}{l}
x_{1, \kappa} \\
x_{2, \kappa}
\end{array}\right)=\left(\begin{array}{c}
\alpha x \\
\beta x
\end{array}\right),
$$


by (5) and since $\left\|J_{\lambda, \kappa} \Phi\left(\begin{array}{l}x_{1, \kappa} \\ x_{2, \kappa}\end{array}\right)\right\| \leq\left\|\Phi\left(\begin{array}{l}x_{1, \kappa} \\ x_{2, \kappa}\end{array}\right)\right\| \underset{\kappa \rightarrow \infty}{\longrightarrow} 0$ by (16). Similarly,

$$
\begin{aligned}
\lim _{\kappa \rightarrow+\infty} \mathcal{A}_{\kappa}\left(\begin{array}{l}
y_{1, \kappa} \\
y_{2, \kappa}
\end{array}\right) & =\left(\begin{array}{l}
A \alpha x \\
A \beta x
\end{array}\right)+\lim _{\kappa \rightarrow+\infty} \mathfrak{A}_{\kappa} J_{\lambda, \kappa} \Phi\left(\begin{array}{l}
x_{1, \kappa} \\
x_{2, \kappa}
\end{array}\right) \\
& =\left(\begin{array}{l}
A \alpha x \\
A \beta x
\end{array}\right)+\lambda \lim _{\kappa \rightarrow+\infty} J_{\lambda, \kappa} \Phi\left(\begin{array}{l}
x_{1, \kappa} \\
x_{2, \kappa}
\end{array}\right)=\left(\begin{array}{l}
A \alpha x \\
A \beta x
\end{array}\right) .
\end{aligned}
$$

\section{Proofs of the main theorems}

\subsection{Proof of Theorem 2.1}

The main tool used in the proof is a singular perturbation theorem of Kurtz [13, 17,18 , which we present here in a simplified form. We start by recalling its basic framework. Suppose, as in Theorem 2.1, that $\mathcal{A}_{\kappa}, \kappa>0$, are generators of semigroups $\left\{\mathrm{e}^{t \mathcal{A}_{\kappa}}, t \geq 0\right\}, \kappa>0$ in a Banach space $\mathbb{B}$, such that (9) holds, and that $Q$ generates a strongly continuous semigroup $\left(\mathrm{e}^{t Q}\right)_{t \geq 0}$ such that

$$
\lim _{t \rightarrow \infty} \mathrm{e}^{t Q} x=: P x, \quad x \in \mathbb{B}
$$

exists. Then $P$ is a bounded idempotent operator and $\operatorname{ker} Q=\operatorname{range} P, \overline{\text { range } Q}=$ ker $P$. Denote:

$$
\mathbb{B}^{\prime}=\text { range } P \text {. }
$$

THEOREM 5.1. (Kurtz) Let $C$ be an operator in $\mathbb{B}, D$ be a subset of its domain, and assume that

(a) for $x \in D$, there are $x_{\kappa} \in D\left(\mathcal{A}_{\kappa}\right)$ such that

$$
\lim _{\kappa \rightarrow+\infty} x_{\kappa}=x \text { and } \lim _{\kappa \rightarrow+\infty} \mathcal{A}_{\kappa} x_{\kappa}=C x
$$

(b) for $y$ in a core $D^{\prime}$ of $Q$, there are $y_{\kappa} \in D\left(\mathcal{A}_{\kappa}\right)$ such that

$$
\lim _{\kappa \rightarrow+\infty} y_{\kappa}=y \text { and } \lim _{\kappa \rightarrow+\infty} \kappa^{-1} \mathcal{A}_{\kappa} x_{\kappa}=Q x,
$$

(c) the operator PC with domain $D \cap \mathbb{B}^{\prime}$ is closable and its closure $\overline{P C}$ generates a strongly continuous semigroup in $\mathbb{B}^{\prime}$.

Then

$$
\lim _{\kappa \rightarrow+\infty} \mathrm{e}^{\mathcal{A}_{\kappa} t} x=\mathrm{e}^{t \overline{P C}} P x, \quad x \in \mathbb{B}, t>0 .
$$

For $x \in \mathbb{B}^{\prime}$, the same is true for $t=0$ as well and the limit is almost uniform in $t \in[0, \infty)$; for other $x$, the limit is almost uniform in $t \in(0, \infty)$. 
In the context of Theorem 2.1, we take $\mathbb{B}=\mathbb{X} \times \mathbb{X}, Q=\mathcal{Q}$, and then (20) follows by (8) with $P=\mathcal{P}$. Also, $\mathbb{B}^{\prime}$ is the space $\mathbb{X}^{\prime}$ of vectors of the form $\left(\begin{array}{l}\alpha x \\ \beta x\end{array}\right), x \in \mathbb{X}$. Let $C$ be the operator in $\mathbb{B}$ given by

$$
C\left(\begin{array}{c}
\alpha x \\
\beta x
\end{array}\right)=\left(\begin{array}{c}
A \alpha x \\
A \beta x
\end{array}\right), \quad D(C)=\left\{\left(\begin{array}{c}
\alpha x \\
\beta x
\end{array}\right) ; x \in D(A)\right\} .
$$

This operator is well defined since we assumed that $\alpha$ leaves $D(A)$ invariant. By Lemma 4.4, condition (a) in Kurtz's theorem is satisfied with $D=\mathcal{I} D\left(A_{\mathrm{a}}\right)$ (the image of $D\left(A_{\mathrm{a}}\right)$ via the isomorphism $\left.\mathcal{I}\right)$. Next, for $x_{1} \in D\left(A_{1}\right), x_{2} \in D\left(A_{2}\right)$, we have $\lim _{\kappa \rightarrow+\infty} \kappa^{-1} \mathcal{A}_{\kappa}\left(\begin{array}{l}x_{1} \\ x_{2}\end{array}\right)=\mathcal{Q}\left(\begin{array}{l}x_{1} \\ x_{2}\end{array}\right)$ so that (b) in Kurtz's theorem is satisfied with $D^{\prime}=D\left(A_{1}\right) \times D\left(A_{2}\right)$. The latter set is a core for $\mathcal{Q}, \mathcal{Q}$ being bounded. Finally, $D \cap \mathbb{B}^{\prime}=D=\mathcal{I} D\left(A_{\mathrm{a}}\right)$ and

$$
P C \mathcal{I} x=P C\left(\begin{array}{c}
\alpha x \\
\beta x
\end{array}\right)=\left(\begin{array}{c}
\alpha A x \\
\beta A x
\end{array}\right)=\mathcal{I} A x=\mathcal{I} A_{\mathrm{a}} x, \quad x \in D\left(A_{\mathrm{a}}\right) .
$$

This shows that $P C$ is an isomorphic copy in $\mathbb{B}^{\prime}$ of the generator $A_{\mathrm{a}}$ in $\mathbb{X}$, and hence is a generator, as well. Therefore, Kurtz's theorem is applicable, and (10) is a particular case of (21).

\subsection{Proof of Theorem 2.2}

Theorem 2.2 is a direct consequence of Theorem 2.1 and the general principle saying that bounded perturbations of semigroups preserve convergence (compare [7]). To explain this in more detail, suppose that $\left(\mathrm{e}^{t \mathcal{A}_{\kappa}}\right)_{t \geq 0}$ are equibounded, strongly continuous semigroups in a Banach space $\mathbb{B}$ converging as $\kappa \rightarrow \infty$ to a semigroup $(T(t))_{t \geq 0}$ which is strongly continuous only on a subspace $\mathbb{B}^{\prime} \subset \mathbb{B}$. Assume also that $\mathcal{B}_{\kappa}$ are bounded linear operators converging strongly to a $\mathcal{B}$, as $\kappa \rightarrow \infty$. Then, by the Phillips perturbation theorem

$$
\mathrm{e}^{t\left(\mathcal{A}_{\kappa}+\mathcal{B}_{\kappa}\right)}=\sum_{n=0}^{\infty} S_{\kappa, n}(t)
$$

where

$$
S_{\kappa, 0}=\mathrm{e}^{t \mathcal{A}_{\kappa}}, \quad S_{\kappa, n+1}(t)=\int_{0}^{t} \mathrm{e}^{(t-s) \mathcal{A}_{\kappa}} B_{\kappa} S_{\kappa, n}(s) \mathrm{d} s, \quad n \geq 0 .
$$

We note that $\left\|S_{\kappa, n}(t)\right\| \leq M \frac{(M K t)^{k}}{k !}$, where $M:=\sup _{\kappa>0, t \geq 0}\left\|\mathrm{e}^{t \mathcal{A}_{\kappa}}\right\|$ and $K:=$ $\sup _{\kappa>0}\left\|\mathcal{B}_{\kappa}\right\|$. Since $S_{\kappa, 0}$ converges by assumption and the Lebesgue dominated convergence theorem, together with the inductive assumption that $\lim _{n \rightarrow \infty} S_{\kappa, n}(t)=$ : $S_{\kappa}(t)$, implies

$$
\lim _{\kappa \rightarrow+\infty} S_{\kappa, n+1}(t)=\int_{0}^{t} T(t-s) \mathcal{B} S_{n}(s) \mathrm{d} s,
$$


all summands in (22) converge. Using the Lebesgue dominated convergence theorem again, we see that

$$
\lim _{\kappa \rightarrow+\infty} \mathrm{e}^{t\left(\mathcal{A}_{\kappa}+\mathcal{B}_{\kappa}\right)}=\sum_{n=0}^{\infty} S_{n}(t)
$$

where $S_{0}(t)=T(t), S_{n+1}(t)=\int_{0}^{t} T(t-s) \mathcal{B} S_{n}(s) \mathrm{d} s, k \geq 0$. Note that the limit is almost uniform in $t \in[0, \infty)$ iff such is the limit of $\mathrm{e}^{\mathcal{A}_{\kappa} t}$.

A special case is obtained for

$$
T(t)=\mathrm{e}^{t G} \mathcal{P},
$$

where $G$ is the generator of a strongly continuous semigroup in $\mathbb{B}^{\prime}$ and $\mathcal{P}$ is an idempotent operator projecting $\mathbb{B}$ onto $\mathbb{B}^{\prime}$. Then,

$$
S_{0}(t)=\mathrm{e}^{t G} \mathcal{P}, \quad S_{n+1}(t)=\int_{0}^{t} \mathrm{e}^{(t-s) G} \mathcal{P B} S_{n}(s) \mathrm{d} s \mathcal{P}, \quad n \geq 0 .
$$

In other words,

$$
\lim _{\kappa \rightarrow+\infty} \mathrm{e}^{t\left(\mathcal{A}_{\kappa}+\mathcal{B}_{\kappa}\right)}=\mathrm{e}^{t(G+\mathcal{P} \mathcal{B})} \mathcal{P},
$$

where $\mathcal{P B}$ is treated as a bounded operator in $\mathbb{B}^{\prime}$.

In the setup of Theorem 2.2, the semigroups $\left(\mathrm{e}^{t \mathcal{A}_{\kappa}}\right)_{t \geq 0}$ are not equibounded, but stability condition (9) allows for reducing the problem to the former case by standard arguments. Moreover, $\mathcal{B}_{\kappa}=\mathcal{B}=\left(\begin{array}{cc}B_{1} & 0 \\ 0 & B_{2}\end{array}\right)$ (by the Phillips perturbation theorem, $\left(\mathrm{e}^{t\left(\mathcal{A}_{\kappa}+\mathcal{B}\right)}\right)_{t \geq 0}$ satisfy the stability condition (9), perhaps with a larger $\left.\omega\right)$. Since the limit in (10) has the form (23) with $G$ being the isomorphic copy of $A_{\mathrm{a}}$ in $\mathbb{X}^{\prime}$, Theorem 2.1, combined with the principle set up above, yields (11), because

$$
\begin{aligned}
\mathcal{P B I x} & =\mathcal{P B}\left(\begin{array}{l}
\alpha x \\
\beta x
\end{array}\right)=\mathcal{P}\left(\begin{array}{l}
B_{1} \alpha x \\
B_{2} \beta x
\end{array}\right)=\left(\begin{array}{l}
\alpha\left(B_{1} \alpha x+B_{2} \beta x\right) \\
\beta\left(B_{1} \alpha x+B_{2} \beta x\right)
\end{array}\right) \\
& =I\left(B_{1} \alpha x+B_{2} \beta x\right), \quad x \in \mathbb{X},
\end{aligned}
$$

i.e., $\mathcal{P B}$ restricted to $\mathbb{X}^{\prime}$ is the isomorphic copy in $\mathbb{X}^{\prime}$ of $B_{1} \alpha+B_{2} \beta$ in $\mathbb{X}$ via the isomorphism $\mathcal{I}$. This completes the proof.

\section{Acknowledgments}

This research was supported by the Polish Government under Grant 6081/B/H03/2011/40, and by the National Research Foundation of South Africa under Grant No. 85406 and the UKZN Research Fund.

Open Access. This article is distributed under the terms of the Creative Commons Attribution License which permits any use, distribution, and reproduction in any medium, provided the original author(s) and the source are credited. 


\section{REFERENCES}

[1] O. Arino, E. Sanchez, R. Bravo de la Parra, and P. Auger, A singular perturbation in an agestructured population, SIAM J. Appl. Math. 60 (1999-2000), no. 2, 408-436.

[2] J. Banasiak and L. Arlotti, Perturbations of positive semigroups with applications, Springer, 2006.

[3] J. Banasiak and A. Goswami, Singularly perturbed population models with reducible migration matrix. 1. Sova-Kurtz theorem and the convergence to the aggregated model, Discr. Cont. Dyn. Syst. A 35 (2015), no. 2, 617-635.

[4] J. Banasiak, A. Goswami, and S. Shindin, Aggregation in age and space structured population models: an asymptotic analysis approach, J. Evol. Equ. 11 (2011), 121-154.

[5] J. Banasiak, A. Goswami, and S. Shindin, Singularly perturbed population models with reducible migration matrix: 2. Asymptotic analysis and numerical simulations, Mediterr. J. Math. 11 (2014), no. 2, 533-559.

[6] J. Banasiak and M. Lachowicz, Methods of Small Parameter in Mathematical Biology, Modeling and Simulation in Science, Engineering and Technology, Birkhäuser, Springer, Heidelberg, 2014.

[7] A. Bobrowski, Degenerate convergence of semigroups related to a model of stochastic gene expression, Semigroup Forum 73 (2006), no. 3, 345-366.

[8] A. Bobrowski, On a semigroup generated by a convex combination of two Feller generators, J. Evol. Equ. 7 (2007), no. 3, 555-565.

[9] A. Bobrowski, Lord Kelvin's method of images in the semigroup theory, Semigroup Forum 81 (2010), 435-445.

[10] A. Bobrowski and R. Bogucki, Semigroups generated by convex combinations of several Feller generators in models of mathematical biology, Studia Mathematica 189 (2008), 287-300.

[11] A. Bobrowski and R. Bogucki, Two theorems on singularly perturbed semigroups with applications to models of applied mathematics, Discr. Cont. Dyn. Syst. B 17 (2012), no. 3, 735-757.

[12] K.-J. Engel and R. Nagel, One-Parameter Semigroups for Linear Evolution Equations, Springer, New York, 2000.

[13] S. N. Ethier and T. G. Kurtz, Markov processes. Characterization and convergence, Wiley, New York, 1986.

[14] F. R. Gantmacher, Applications of the theory of matrices, Interscience Publishers, Inc. New York, 1959.

[15] G. Greiner, Perturbing the boundary conditions of a generator, Houston J. of Mathematics 13 (1987), no. 2, 213-229.

[16] M. Iannelli, Mathematical theory of age-structured population dynamics, Applied Mathematical Monographs 7, Giardini Editori E Stampatori, Pisa, 1995.

[17] T.G. Kurtz, A limit theorem for perturbed operator semigroups with applications to random evolutions, J. Functional Analysis 12 (1973), 55-67.

[18] T.G. Kurtz, Applications of an abstract perturbation theorem to ordinary differential equations, Houston J. Math. 3 (1977), no. 1, 67-82.

[19] J.D. Murray, Mathematical biology, Springer, 1993, Second, corrected edition.

[20] E. Sanchez, R. Bravo de la Parra, P. Auger, and P. Gomez-Mourelo, Time scales in linear delayed differential equations, J. Math. Anal. Appl. 323 (2006), 680-699.

[21] G. F. Webb, Theory of nonlinear age-dependent population dynamics, Theory of Nonlinear AgeDependent Population Dynamics, 1985.

[22] G. F. Webb, Structured population dynamics, Mathematical Modelling of Population Dynamics, R. Rudnicki ed., Banach Center Publications 63, 2004, pp. 123-163. 


\section{J. Banasiak}

School of Mathematical Sciences, University of KwaZulu-Natal, Durban, South Africa and

Institute of Mathematics,

Technical University of Łódź, Łódź, Poland

E-mail:banasiak@ukzn.ac.za

A. Bobrowski

Lublin University of Technology, Lublin, Poland

E-mail: a.bobrowski@pollub.pl 